\title{
Artificial intelligence in corneal topography
}

\author{
Nazar Saleh* ${ }^{*}$, Nebras Hussein ${ }^{1}$ \\ ${ }^{1}$ Khwarizmi College of Engineering, Baghdad, Iraq.
}

\begin{abstract}
The purpose of this paper is to explore the effectiveness and efficiency of various artificial intelligence (AI) techniques in extracting features from corneal topographies. A considerable number of dated and contemporary related research papers have been reviewed. The author has only checked the studies that considered developing at least one AI-based algorithm for data classification of topographic patterns. The results of this review emphasize the effectiveness and efficiency of machine learning algorithms in the clinical diagnosis of various eye refractive problems.
\end{abstract}

Keywords: Artificial intelligence, classification, feature extraction, topographic images.

\section{Korneal topografide yapay zeka}

Öz

Bu yazının amacı kornea topografyasından özelliklerin çıkarılmasında çeşitli yapay zeka tekniklerinin etkinliğini ve etkinliğini araştırmaktır. Önemli sayıda çağdaş ve güncel araştırma makaleleri gözden geçirilmiştir. Yazar, sadece topografik modellerin veri sınıflandırması için en az bir AI tabanlı algoritmanın geliştirilmesini düşündüğü çalışmaları kontrol etmiştir. Bu derlemenin sonuçları, çeşitli göz kırıcı problemlerinin klinik tanısında, makine öğrenimi algoritmalarının etkinliğini ve verimliliğini vurgulamaktadır.

Anahtar Kelimeler: Yapay zeka, sınıflandırma, özellik çıkarma, topografik görüntüler.

\section{Introduction}

Topographical images of the human cornea anterior and posterior surfaces are becoming more important diagnostic tools than ever in the classification of the healthy and unhealthy corneas. These maps are created by a system called Pentacam. The final maps of this system carry a lot of details that help and guide the medical decision. Finding hidden details and reporting the expected clinical situation is in most of the time, related strongly to the experience and level of knowledge of the final decision maker (ophthalmologist).

At the same time, the artificial intelligence started to play the main role in the medical imaging analysis and smart diagnosis. These tools accompanied by the huge development level encountered in the computer (software and hardware) science introduce a new analysis area and unprecedented tools. The capability of recognizing particular features of these maps and defining the clinical interrelationship among them plays new roles in the artificial intelligence of the medical imaging field. These selected features are learned and specified earlier through a number of cases that have been pre-diagnosed and reported by ophthalmologists.

\subsection{Cornea}

The cornea is the outlying part of the human eye and holds a significant focusing power. It is of a domed shape and transparent in nature. Despite this transparency, the cornea is characterized by a perfect organization of tissues and with neither substances nor blood vessels. It gets its nourishment and infections protection aspect from two other parts, the fluid layer that resides behind it and called the aqueous humans and the tears (Camarillo et al., 2002).

\subsection{Corneal diseases}

It is significant to interpret the biomechanical response of cornea for post-surgery cases and some diseases. The cornea is the topmost lens of the eye and accountable for $65-75 \%$ of the sight strength and therefore any disorder in the cornea curvature results in a sight disorder or refractive problems including, astigmatism, hyperopia, and myopia. High corneal 
curvature leads to nearsightedness or myopia. Low corneal curvature causes farsightedness or hyperopia [2]. These two refractive diseases may also occur due to a malfunction of the lens. Additionally, uneven or abnormal corneal curvature causes blurred vision or astigmatism [3].

\subsection{Pentacam}

Pentacam is a powerful scanner for the eye segments and based on the Scheimpflug camera measurement. It is a robust invention that helps ophthalmologists in investigating the corneal anterior surface. Although Scheimpflug technique was known for scanning anterior eye segments since the 70 s, it was not before the $80 \mathrm{~s}$ and $90 \mathrm{~s}$ that it was released commercially. In 2005, it started to be used widely clinically [4].

\subsection{Corneal topographic maps}

The four major topographic patterns are the pachymetry pattern, the anterior curvature sagittal pattern, in addition to both the anterior and posterior elevation pattern (Mazen M Sinjab, 2015) ( as indicated in Fig.1). These patterns reflect lots of details about the anterior eye surface which should be tested by the specialist.

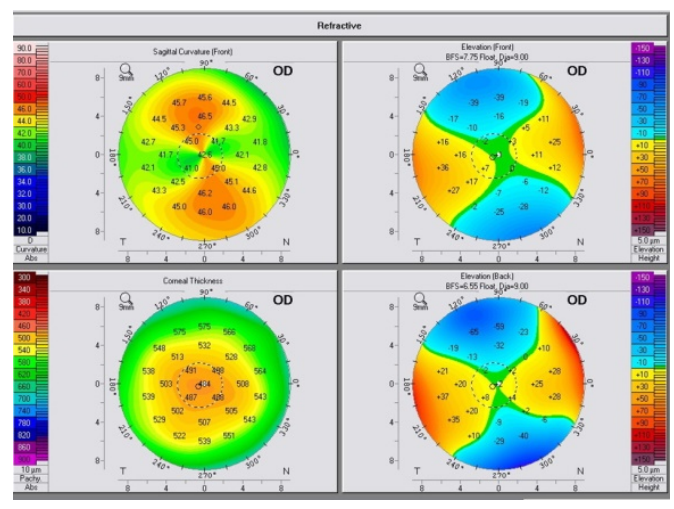

Figure 1. The four refractive topographical map

\section{Artificial intelligence techniques}

\subsection{Artificial Neural Networks (ANNs) Based Classifiers}

Some researchers, as early as 1995 , attempted to evaluate the capabilities and usefulness of an automated system based on ANNs in interpreting the corneal topographic maps. They asserted that the difficulty of corneal topographic pattern interpretation, specifically when the topographic maps are similar to other pathologies, encouraged them to investigate the capabilities of an automated approach for diagnosing shape abnormalities in the cornea. The study reported that the ANNs system may need further enhancements to be an objective computerized classification tool of video-keratography for helping the doctors in determining the abnormalities in corneal topographies (Maeda, Klyce, \& Smolek, 1995).

Smolek and Klyce (1997) conducted a study to compare four video-keratographic based keratoconus detection methods to a new detection method based on ANNs. The researchers designed a neural network approach that was able to classify the keratoconus screening to automatically discover whether the keratoconus suspected (KCS) or keratoconus (KC) exists. The research findings assured that the ANNs approach succeeded in differentiating between the topographies of the $\mathrm{KC}$ from $\mathrm{KCS}$, and even from other cases that bear a resemblance to KC. Moreover, the outcomes proved that the performance of the new ANNs detection method outweighed the other detection methods that the researcher examined in the study. The ANNs method recorded a higher performance in terms of specificity and accuracy. However, the researchers demonstrated that the sensitivity of all the detection methods including the ANNs was equal (Smolek \& Klyce, 1997).

In 2008, for classifying particular types of corneal shapes, a study conducted to develop and compare two different classification techniques, the discriminant analysis, and ANNs, provided that the Zernike coefficients are given. It is noted that just a few studies have applied Zernike coefficients (ZC) as input parameters (Carvalho, 2005; Schwiegerling \& Greivenkamp, 1996).

Despite the fact that the researchers used a comparatively small database, the study's outcomes confirm that for automated diagnosis of videokeratography patterns by the ANNs or discriminant analysis, a possible reliable parameter may be the Zernike coefficients to be used as input data as descriptors of the corneal shape (de Carvalho \& Barbosa, 2008).

In 2018, some researchers, at Shiley Eye Institute at UC San Diego Health and University of California San Diego School of Medicine, have developed a new ANNs-based system to examine patients with common retinal diseases that cause blinding but are treatable. The diagnosis of both the macular degeneration related to age and diabetic macular edema was comparable to the experts' classification. They additionally indicated that the automated system introduced more interpretable and transparent diagnosis because it was able to determine the regions that the ANNs identified (Kermany et al., 2018; LaFee, 2018).

\subsection{Support Vector Machines (SMVs) Based Classifiers}

In 2005, a researcher developed a framework built on SMVs for ophthalmology pattern interpretation. 
And to assist optometry doctors in the diagnosis of ocular refraction problems including short-sightedness, hypermetropia, and astigmatism. The outcomes of the project recommended a method for improving the understanding of eye patterns that were obtained from a system called HS (Hartmann-Shack), provided that other ocular errors measurements are measured and detected. The system was designed to interpret and analyze eye patterns as a whole without the need of having a reference pattern to evaluate the difference between the obtained data between the target and reference pattern (Netto, 2005).

In 2008, some researchers attempted to perform a classification of corneal topographies using the SVM technology. The study conducted to prove the success of a learning algorithm uses the SVM technology in distinguishing between corneal diseases such as keratoconus, suspect keratoconus, hyperopic and myopic laser vision correction, orthokeratology, and pellucid marginal degeneration from normal corneal conditions. The researchers pursued their study through testing topographies obtained by the Corneal Topographer ATLAS ${ }^{\circledR}$ Model 90000. The algorithm was trained and evaluated using previous data on 239 abnormal corneal cases and 85 normal cases. For the training purpose, four-fifths of the cases were selected randomly, while the remaining fifth was used for the evaluation purpose. The accuracy, sensitivity, and specificity of the algorithm were equal to or greater than $90 \%$. The SVM-based model was able to identify the cases of abnormal corneas from normal conditions using the randomly picked training data (Bagherinia et al., 2008).

In 2012, a study developed an SVM-based algorithm for subclinical keratoconus and keratoconus diagnosis through tomography and topography data. It introduced a new data classifier algorithm for the detection of keratoconus using measurements of corneas obtained by Placido corneal topography together with a Scheimpflug camera. The SVM demonstrated a high specificity, sensitivity, and accuracy, in distinguishing four eye conditions, eyes with previous surgery history, eyes with subclinical keratoconus or keratoconus, and normal eyes. The algorithm accuracy was excellent for the cases regardless of if it was provided with the data obtained from the corneal thickness and posterior corneal surface or not. The rate of valid predictions was $>95 \%$ when provided with additional data, and $93 \%$ when no additional data was provided. It is obvious that when additional data of the posterior corneal surface was provided, the precision was higher. The pachymetry and the posterior and anterior corneal surfaces additional data increased the sensitivity of the classifier (Arbelaez, Versaci, Vestri, Barboni, \& Savini, 2012).

A study developed a new diagnosis system for vision-related problems. It presented a system that is capable of performing two tasks, first to diagnose the Short-Sightedness (Myopia ) or Long-Sightedness
(Hypermetropia ) and second to identify the measurements of SPH (eyesight distance) and CYL (deviation). The researchers believe that it is easy for a physician to diagnose the problem if the SPH or CYL lies within the normal measurements, however, when any of them lies out of normal figures then it is not such an easy task to detect and it takes more time for the doctor to determine the condition. Therefore, they proposed a model based on SVM and ANNs to detect eyesight problems and to help doctors decide appropriately and quickly. The ANNs is applied due to its known strength in checking and classifying sightedness of the eye. The developers trained the ANNs by using Backpropagation algorithm due to its high capability in maps classification especially nonlinear mapping. The results showed that with ANNs, the nonlinear SVMs specificity increased from $92 \%$ to $97 \%$. These results prove the capabilities of ANNs as well as SVM as pattern recognition techniques (Kotsia \& Pitas, 2007; Pontil \& Verri, 1998) in detecting the state of the eyesight (Noaman, Muharram, \& Alqubati, 2014).

A study conducted to meet two purposes; first is to test the reliability of an SVM-based algorithm for detecting corneal patterns objectively and automatically using a set of twenty-two parameters provided by Pentacam measurements, and second is to put the algorithm model outcomes in comparison with other popular keratoconus classifiers results. For the classification, 22 parameters were applied to an SVM algorithm developed as a piece of computer software based on machine-learning and called Weka. The researchers calculated and compared the accuracy through cross-validation tests for, $\mathrm{KC}$ versus normal, forme fruste versus normal and across all the 5 cohorts, with other outputs of some other popular classification models. In case of the $\mathrm{KC}$ vs. normal, the algorithm outstanding accuracy was $98.9 \%$, while $99.1 \%$ recorded for sensitivity, and $98.5 \%$ obtained for the $\mathrm{KC}$ detection specificity. For the forme fruste versus normal eyes case, the results were $93.1 \%$ for accuracy, $79.1 \%$ for sensitivity, and $97.9 \%$ for specificity. For the classification of the five cohorts, $88.8 \%$ was recorded for accuracy and $89.0 \%$ for the average sensitivity, and $95.2 \%$ for specificity (Ruiz Hidalgo et al., 2016).

\subsection{Decision Tree - Based Classifiers}

In 2005, a study applied an automated classification system based on machine learning and decision tree induction to distinguish between keratoconus and normal corneal shapes quantitatively and objectively. They also set a comparison between the developed classifier and other popular classification systems. A model of seventh-order Zernike polynomial was applied to the corneal surface. The classifier was based on the C4.5 algorithm for the DT classifier. The outcomes of the automated classifier were compared 
with several modified indices, the KISA\%, the Cone Location and Magnitude, Keratoconus Prediction Index (KPI), Schwiegerling's Z3, and RabinowitzMcDonnell- given that the standard thresholds of classification for every method were followed. For every method of classification, the researcher additionally examined the area under the ROC (receiver operator characteristic) curve. The performance of the developed classification method based on DT was greater than or equal to the other classification methods examined. The results showed $92 \%$ for accuracy, and 0.97 for the area under the curve of ROC. With 4 of 36 surface features of Zernike polynomial coefficients, the automated DT model decreased the required information for the discrimination between keratoconus and normal eye. The 4 classification attributes the study used were greater sagittal depth, inferior elevation, trefoil and oblique toricity. The research findings confirmed the capability of the decision tree-based classifier in distinguishing among various corneal shapes, it is also assured that it is a dependable quantitative classifier through Zernike polynomials and any device which can produce raw elevation data. They also assured that the framework is applicable to other problems of classification (Twa et al., 2005).

Kabari and Nwachukwu (2012) introduced a framework combining ANNs and DTs for various eyes diseases diagnosis such as myopia, hyperopia, and Astigmatism. It is distinguished from other related studies because it didn't exploit one algorithm only but two technologies are used for data mining ANNs and DTs. The researchers presented a hybrid model called NNDTEDDS (Neural Networks Decision Trees Eye Disease Diagnosing System). They asserted that both technologies have been tools for discovering knowledge and introducing a hybrid model, the researchers developed rules that show how eye diseases diagnosis is done based on the physical conditions of the eye and its related symptoms. These rules show how the knowledge obtained in neural networks after being trained from earlier samples of physical conditions of eye and symptoms. The findings of the study prove a considerable success of $92 \%$, which reflects how combining the two technologies are efficient and effective in diagnosing eye diseases such as myopia, hyperopia, and astigmatism. Moreover, Kabari and Nwachukwu assured that the concluded rules are useful in teaching younger ophthalmologists (Kabari \& Nwachukwu, 2012)

\subsection{Naive Bayes Based Classifiers}

An experimental research introduced an automated, Naive Bayes-based system for detecting eye disease. The automated system uses CBR (Case-Based Reasoning) as an experience-based model for reasoning, and the Naïve Bayes as an eye diseases classifier by adopting the theorem of Bayes. The findings showed that the accuracy of the automated model of Naïve Bayes was $82 \%$. Accordingly, they concluded that an expert system based on a combination of Naïve Bayes and CBR can be a promising technology for eye diseases diagnosis. Naïve Bayes classifier is capable of introducing effective diagnoses for people but more improvements and enhancements were still required (Kurniawan, Yanti, \& Ahmad Nazri, 2014).

\subsection{Studies comprised Several Classifiers}

The focus of a study conducted in 2012 was to examine the effectiveness of an AI-based system in Keratoconus diagnosis. The researcher believed that the developed system would help device producers to enhance their production so that the medical system can assist experts and support the recognition phase automatically. They trained some classifiers, DT, SVM, ANNs, RBFNN, and Multi-Layer-Perceptron to recognize whether it is a Keratoconus or suspect to Keratoconus eye. The classifiers were trained with part of the dataset and tested by the rest. The output of the study indicated that the proposed algorithm 's accuracy was $91 \%$ in discrimination among $\mathrm{KC}$, suspect to $\mathrm{KC}$ and normal eye. This accuracy assures the capability of the developed algorithm in automatic detection of $\mathrm{KC}$ or suspects to KC (Toutounchian, Shanbehzadeh, Khanlari, \& Stage, 2012).

In 2014, a survey conducted to review several automated, computer-based systems for ocular diseases diagnosis. The researchers focused on three kinds of data comprising imaging, clinical, and genetic. According to the researchers, such types of data were the commonly used data types in the computer-aided diagnosis for ocular diseases such as Pathological Myopia, Macular Degeneration that is age-related, Glaucoma, and Diabetic Retinopathy. The researchers asserted that over the past years, ocular diseases diagnosis that is based on computer-aided models has shown substantial advancement. However, the significance of having fully automatic models which are capable of exploiting the clinical knowledge and incorporate mixed data sources still needed for future development (Zhang et al., 2014).

\subsection{Google`s DeepMind Health Project}

AI is extensively applied for tracing, normalizing, collecting, and storing data in healthcare. In this regard, Google`s AI research branch announced its new project to investigate the extent to which technology could be efficient in analyzing eye scans through medical records data mining for the purposes of delivering faster and better health services and improving eye treatment. The project is called DeepMind Health. It is a cooperative project with Moorfields Eye Hospital NHS Foundation Trust launched in 2016. Although the project is still in the early stages of AI research in health, Deep Mind Health reassures that the undertaken 
research will have practical benefits, and the outcomes of the study will be submitted to academic journals that are peer-reviewed after performing the strict clinical scrutiny ("Researching for tomorrow," 2018).

\section{Conclusion}

It is beneficial and helpful to explore the plenty of attempts of applying AI to eye diseases diagnosis throughout the history. The exploration of related works over the past decades gives some insights as to what extent the AI-based technology supports the medical care in areas where much effort and time are required by specialists. Numerous studies have been conducted to test the accuracy and specificity of one or more of the artificial intelligence algorithms such as the ANNs, SVMs, DT, and Naive Bayes. Some researchers have tested one algorithm while others have concerned with combining two or more algorithms. All the reviewed research reported high efficiency and effectiveness of the tested algorithms.

\section{References}

Arbelaez, M. C., Versaci, F., Vestri, G., Barboni, P., \& Savini, G. (2012). Use of a support vector machine for keratoconus and subclinical keratoconus detection by topographic and tomographic data. Ophthalmology, 119(11), 2231-2238.

https://doi.org/10.1016/j.ophtha.2012.06.005

Bagherinia, H., Chen, X., Flachenecker, C., Angeles, R., Burger, D., Caroline, P., ... Reeder, K. (2008). Support Vector Machine (SVM)-Based Classification of Corneal Topography. Investigative Ophthalmology \& Visual Science, 49(13), 1023. Retrieved from http://dx.doi.org/

Camarillo, T., Choi, K., Hamilton, G., Miles, M., Muller, K., Williams, K., ... Schrepel, P. (2002). Athletes as an Ideal Target Population for Orthokeratology Keratoconus : Improving Quality of Life Through Advancements in Detection and Treatment.

Carvalho, L. A. (2005). Preliminary Results of Neural Networks and Zernike Polynomials for Classification of Videokeratography Maps: Optometry and Vision Science, 82(2), 151-158. https://doi.org/10.1097/01.OPX.0000153193.415 54.A1

de Carvalho, L. A., \& Barbosa, M. S. (2008). Neural networks and statistical analysis for classification of corneal videokeratography maps based on Zernike coefficients: a quantitative comparison. Arquivos Brasileiros de Oftalmologia, 71(3), 337-341. Retrieved from http://www.ncbi.nlm.nih.gov/pubmed/18641817

Kabari, L., \& Nwachukwu, E. (2012). Neural Networks and Decision Trees For Eye Diseases Diagnosis. In P. Vizureanu (Ed.), Advances in Expert Systems. InTech.

Kermany, D. S., Goldbaum, M., Cai, W., Valentim, C. C. S., Liang, H., Baxter, S. L., ... Zhang, K. (2018). Identifying Medical Diagnoses and Treatable Diseases by Image-Based Deep Learning. Cell, 172(5), 1122-1131.e9. https://doi.org/10.1016/j.cell.2018.02.010

Kotsia, I., \& Pitas, I. (2007). Facial Expression Recognition in Image Sequences Using Geometric Deformation Features and Support Vector Machines. IEEE Transactions on Image Processing, 16(1), 172-187. https://doi.org/10.1109/TIP.2006.884954

Kurniawan, R., Yanti, N., \& Ahmad Nazri, M. Z. (2014). Expert systems for self-diagnosing of eye diseases using Naïve Bayes (pp. 113-116). IEEE. https://doi.org/10.1109/ICAICTA.2014.7005925

LaFee, S. (2018, February). Artificial Intelligence Quickly and Accurately Diagnoses Eye Diseases and Pneumonia.

Maeda, N., Klyce, S. D., \& Smolek, M. K. (1995). Neural network classification of corneal topography. Preliminary demonstration. Investigative Ophthalmology \& Visual Science, 36(7), 1327-1335.

Mazen M Sinjab. (2015). step by step Reading Pentacam Topography (basics and case study series).

Netto, A. V. (2005). System Based on Computational Intelligence for Ophthalmology Image Understanding. IEEE Latin America Transactions, 3(5), 14-22. https://doi.org/10.1109/TLA.2005.1642434

Noaman, K. M., Muharram, A. A., \& Alqubati, I. A. (2014). Diagnosis of Poor Eyesight based on Support Vector Machine and Artificial Neural Networks. Journal of Emerging Trends in Computing and Information Sciences, 5(10).

Pontil, M., \& Verri, A. (1998). Support vector machines for 3D object recognition. IEEE Transactions on Pattern Analysis and Machine Intelligence, 20(6), 637-646. https://doi.org/10.1109/34.683777

Researching for tomorrow [WWW Document], 2018. DeepMind.

Ruiz Hidalgo, I., Rodriguez, P., Rozema, J. J., Ni Dhubhghaill, S., Zakaria, N., Tassignon, M.-J., \& Koppen, C. (2016). Evaluation of a MachineLearning Classifier for Keratoconus Detection Based on Scheimpflug Tomography. Cornea, 35(6), 827-832. https://doi.org/10.1097/ICO.0000000000000834

Schwiegerling, J., \& Greivenkamp, J. E. (1996). Keratoconus detection based on 
videokeratoscopic height data. Optometry and Vision Science: Official Publication of the American Academy of Optometry, 73(12), 721728.

Smolek, M. K., \& Klyce, S. D. (1997). Current keratoconus detection methods compared with a neural network approach. Invest Ophthalmol.Vis.Sci., 38(0146-0404 (Print)), 2290-2299.

Toutounchian, F., Shanbehzadeh, J., Khanlari, M., \& Stage, A. R. (2012). Detection of Keratoconus and Suspect Keratoconus by Machine Vision. International Multiconference of Engineers and Computer Scientists, 1(March 2012), 14-16.

Twa, M. D., Parthasarathy, S., Roberts, C., Mahmoud, A. M., Raasch, T. W., \& Bullimore, M. A. (2005). Automated Decision Tree Classification of Corneal Shape: Optometry and Vision Science, 82(12), 1038-1046. https://doi.org/10.1097/01.opx.0000192350.0104 $5.6 \mathrm{f}$

Zhang, Z., Srivastava, R., Liu, H., Chen, X., Duan, L., Kee Wong, D. W., ... Liu, J. (2014). A survey on computer aided diagnosis for ocular diseases. BMC Medical Informatics and Decision Making, 14. https://doi.org/10.1186/1472-6947-14-80 